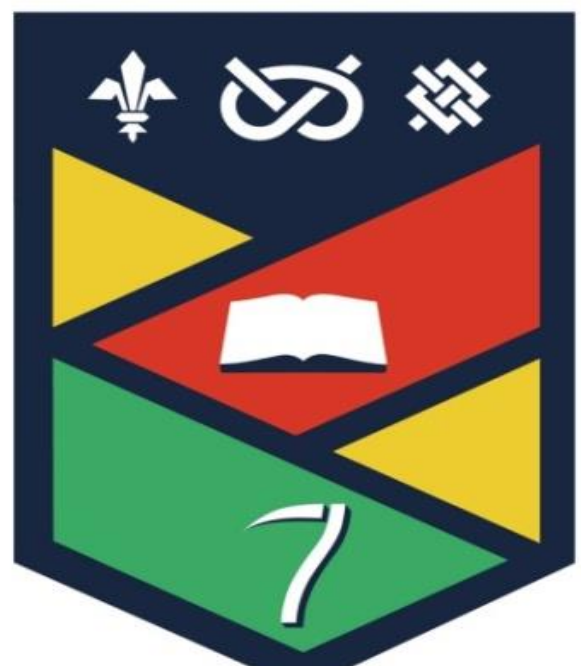

Keele University

\section{Survey on management of post thrombolysis symptomatic intra- cerebral haemorrhage}

University Hospitals Nis of North Midalands

NHS Trust

\section{RESULTS}

There were 64 responses in total with the majority from stroke physicians. The responses were mostly from Europe. The three most common risk factors identified for SICH following thrombolysis were stroke severity, uncontrolled hypertension and previous anti-platelets and/or anti-thrombotics use $(45 \%, 40 \%$ and $16 \%$ respectively). Thrombolytic therapy, antiplatelet therapy and diabetes mellitus were found to be the least contributing risk factors $(11.6 \%, 2.3 \%$ and $0 \%$ respectively). The SICH incident in most hospitals was between $3-6 \%$. The most commonly used management included fresh frozen plasma $(41 \%)$ and cryoprecipitate $(24 \%)$. Less commonly used treatment included vitamin $\mathrm{K}(9 \%)$, aminocaproic acid $(6 \%)$, tranexamic acid $(6 \%)$ and platelet transfusion $(6 \%)$. However the majority of the individual
answers highlighted that no particular management option yielded the best result, but all had similar outcomes. $75 \%$ of the respondent felt that the $\mathrm{SICH}$ associated mortality was $<50 \%$ whilst $20 \%$ found mortality around $80 \%$.

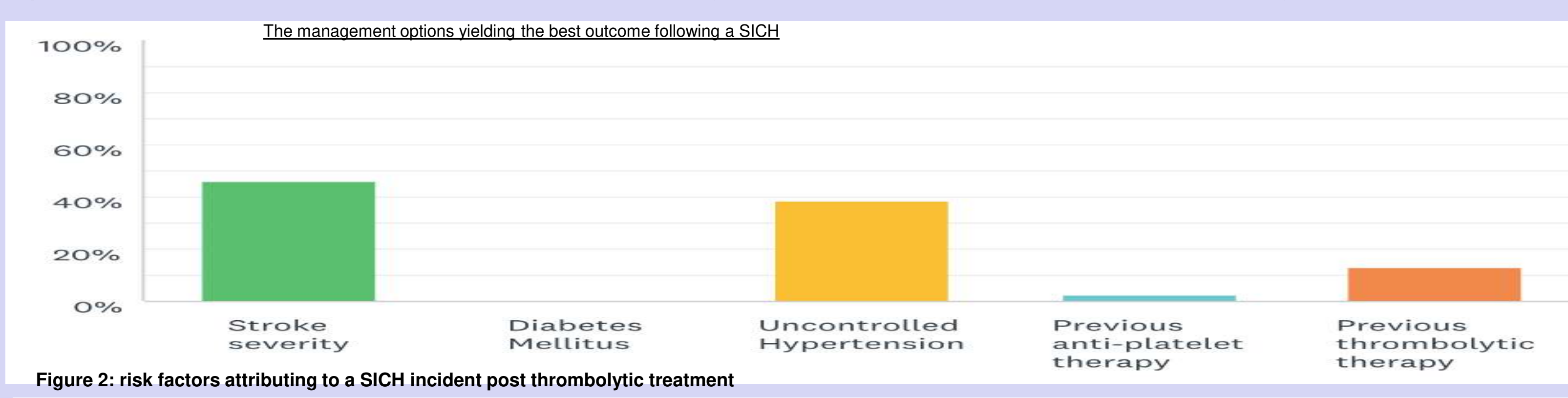

A 12-question survey was devised using SurveyMonkey ${ }^{\circledR}$ (figure 1). The survey was distributed electronically to members of the European Stoke Association (ESO) and World Stroke Organisation (WSO) in December 2017 and remained open for 12 days. Multiple-choice format was primarily used as question style and free text boxes were also used where qualitative comments to open-ended questions were analysed through thematic analysis.

$$
\text { Survey questions }
$$

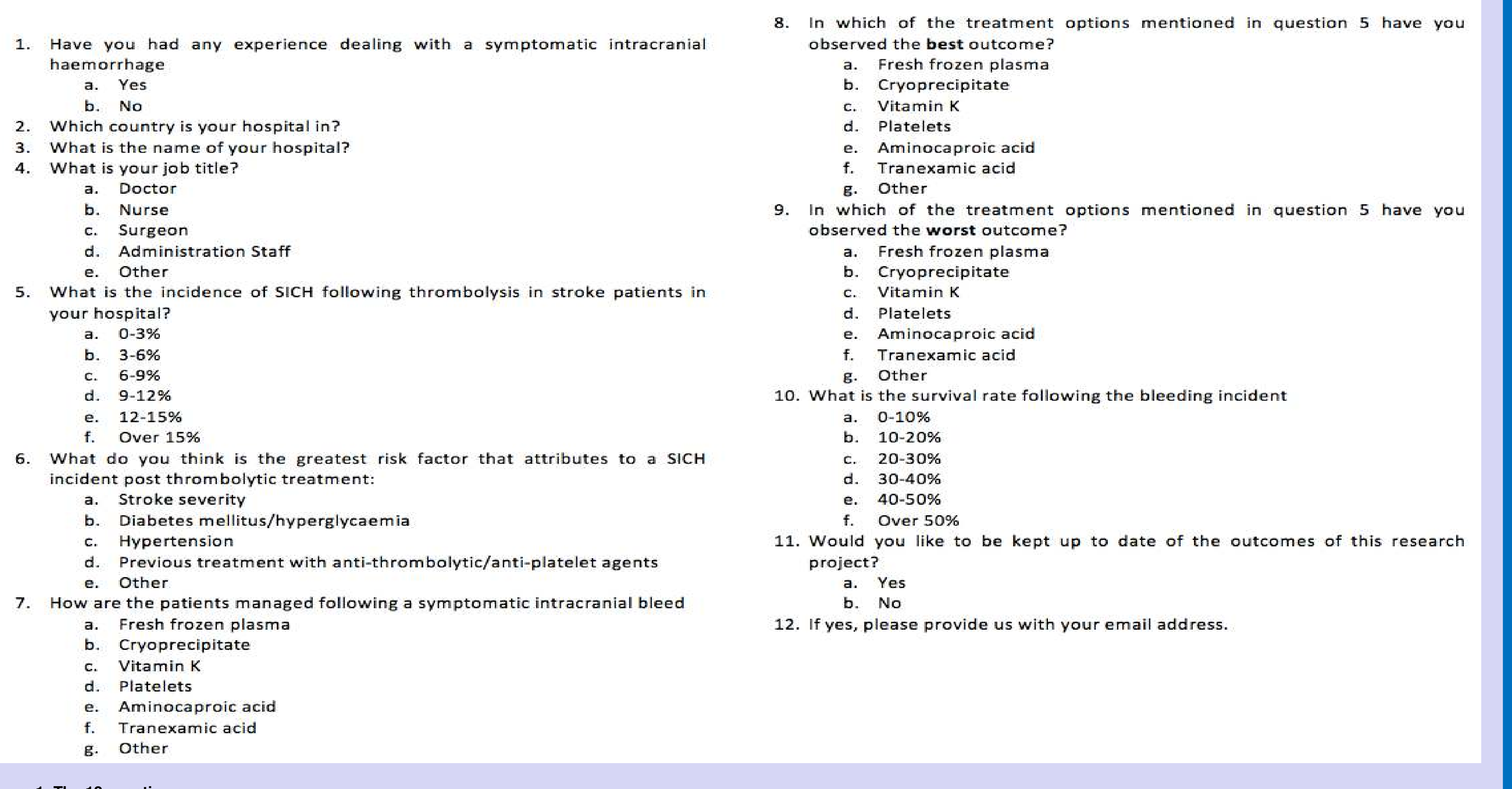

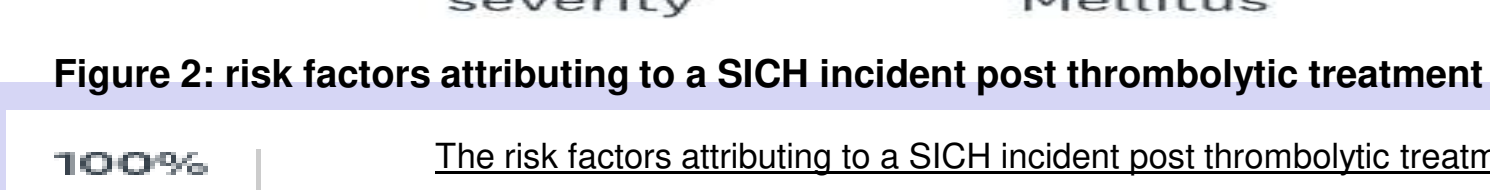

$80 \%$

$60 \%$
$40 \%$
$20 \%$
$0 \%$

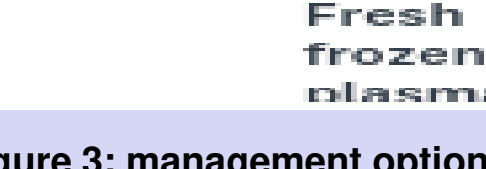

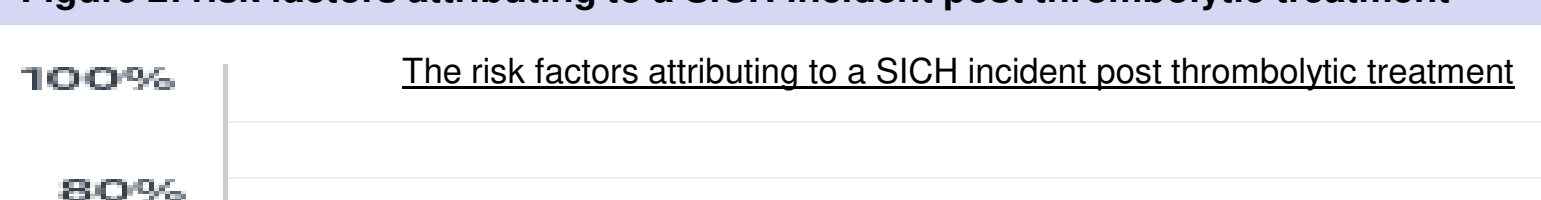

\section{(a)}

\section{CONCLUSIONS}

$>$ This study found that the greatest risk factor leading to SICH was the stroke severity.

- Although fresh frozen plasma yielded the best management option for some respondents, many respondents believed

that all management options have a similar outcome.

> Overall all the respondents agree that $\mathrm{SICH}$ associated mortality is very high.

- Urgent research should be undertaken with newer agents to improve the outcome of the patients.

REFERENCES

Echouffo-Tcheugui $J, W$

2013;:11 (5):862-871.
Mckinney J, Cucchiara B. Risk Scores for Predicting Post-thrombolysis Intracerebral Hemorrhage. US Neurology. 2010:05(02):39.
Oh D, Tham C, Tu T. Pre-existing antiplateletet treatment increases risk of intracerebral haemorrhage in post-thrombolysis acute isch

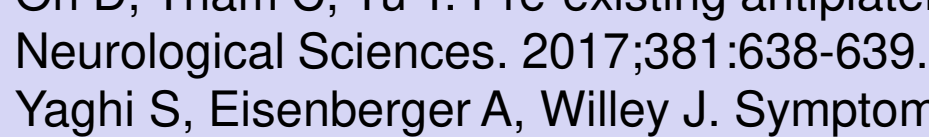
S Eisenberger A Wil Jy Symptomatic Intracero

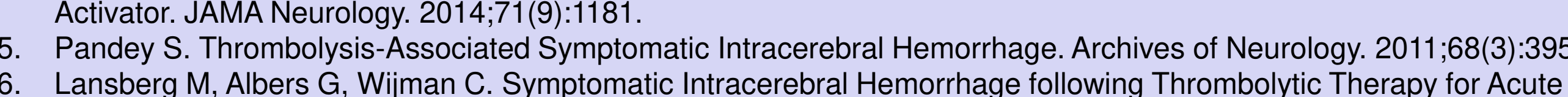

M, Albers G, Wimman C. Symptomatic Intrata Hatten B. Risk Factors of Symptomatici Intracereebral Hemorrhage After tPA Therapy for Acute Stroke. The Journal of Emergency Medicine. 2007;33(4):446-447. 